\title{
Red blood cell distribution width (RDW) is an independent predictor of post-implantation syndrome in patients undergoing endovascular aortic repair for abdominal aortic aneurysm
}

\author{
Gian Franco Veraldi ${ }^{1}$, Luca Mezzetto ${ }^{1}$, Lorenzo Scorsone ${ }^{1}$, Marco Macrì ${ }^{1}$, Chiara Bovo ${ }^{2}$, Fabio \\ Simoncini ${ }^{1}$, Paolo Criscenti ${ }^{1}$, Giuseppe Lippi ${ }^{3}$ \\ ${ }^{1}$ Department of Vascular Surgery, University of Verona-School of Medicine, University Hospital of Verona, Verona, Italy; ${ }^{2}$ Medical Direction, \\ University Hospital of Verona, Verona, Italy; ${ }^{3}$ Section of Clinical Biochemistry, University of Verona, Verona, Italy \\ Contributions: (I) Conception and design: GF Veraldi, C Bovo, G Lippi, (II) Administrative support: None; (III) Provision of study materials or \\ patients: L Mezzetto, L Scorsone, M Macrì, F Simoncini, P Criscenti; (IV) Collection and assembly of data: G Lippi, L Mezzetto, L Scorsone; \\ (V) Data analysis and interpretation: G Lippi; (VI) Manuscript writing: All authors; (VII) Final approval of manuscript: All authors. \\ Correspondence to: Prof. Giuseppe Lippi. Section of Clinical Biochemistry, University Hospital of Verona, Piazzale LA Scuro, 37134 Verona, Italy. \\ Email: giuseppe.lippi@univr.it.
}

Background: This observational, retrospective study aims establishing the role of red blood cell distribution width (RDW) for identifying abdominal aortic aneurism (AAA) patients at risk of developing post-implantation syndrome (PiS) after endovascular aneurysm repair (EVAR).

Methods: The study population consisted of all patients undergoing EVAR for AAA at the University Hospital of Verona (Italy), between June 1, 2016 and May 31, 2018. Blood samples for measuring hemoglobin, mean corpuscular volume (MCV) and RDW were collected at hospital admission and the day after EVAR. The primary endpoint was PiS development. Delta variations were calculated as the ratio between values measured after and before EVAR.

Results: The final study population consisted of 124 patients (10 women and 114 men; median age, 75 years), 55 of whom developed PiS. In patients with or without PiS hemoglobin significantly decreased after EVAR, whilst RDW significantly increased in patients with PiS and decreased in those without. Age, sex, hypertension, diabetes and renal failure were similar in patients who developed PiS or not, whilst a positive history of coronary artery disease was more frequent in PiS patients. Although hemoglobin and MCV changes after EVAR did not differ in patients with or without PiS, delta RDW was higher in those with PiS. The rate of patients with delta $\mathrm{RDW}>1$ was significantly higher in patients with PiS that in those without (61.8\% vs. 34.8\%; $\mathrm{P}=0.002)$. In multivariate analysis, delta RDW remained independently associated with PiS ( $\beta$ coefficient, 2.023; $\mathrm{P}=0.001$ ). A delta $\mathrm{RDW}>1$ after EVAR was associated with $\sim 3$-fold enhanced risk of PiS (odds ratio, 3.04; $\mathrm{P}=0.003$ ) and exhibited a good prognostic performance (area under the curve, $0.69 ; \mathrm{P}<0.001)$.

Conclusions: Calculation of delta RDW after EVAR seems an efficient prognostic tool for stratifying the risk of developing PiS, especially in the early postoperative period.

Keywords: Abdominal aortic aneurism (AAA); endovascular aneurysm repair (EVAR); complications; red blood cell distribution width (RDW)

Submitted Oct 15, 2018. Accepted for publication Nov 01, 2018.

doi: $10.21037 / \mathrm{atm} .2018 .11 .07$

View this article at: http://dx.doi.org/10.21037/atm.2018.11.07 


\section{Introduction}

Abdominal aortic aneurysm (AAA) is conventionally defined as a focal dilation in the lower part of the aorta, displaying a diameter of not less than 1.5 -fold the normal aorta diameter at the level of renal arteries (i.e., $>3 \mathrm{~cm}$ ) (1). The vast majority of AAA (i.e., up to $80 \%$ ) occur in the segment comprised between renal arteries and aortic bifurcation (1). The most frequent underlying causes of AAA include atherosclerosis, hypertension, infections, arteritis and vasculites, disorders of connective tissue (e.g., Marfan and Ehlers-Danlos syndromes), genetic abnormalities and traumas, among others (2). Recent epidemiological data suggests that AAA represents an important cause of morbidity and mortality worldwide, accounting for over 5,000 deaths in the United States. Most deaths are attributable to AAA rupture, which is the most severe complication of this pathology, and whose risk increases from $<5 \%$ for AAA with diameter $<5 \mathrm{~cm}$ to approximately $50 \%$ for those with diameter $>8 \mathrm{~cm}$ (3).

Surgical management is the recommended approach for symptomatic AAA, as well as for patients with AAA diameter larger than $5.5 \mathrm{~cm}(4,5)$. Endovascular aneurysm repair (EVAR) is now regarded as the standard technique for treatment of infrarenal AAA. Although the longterm outcome remains controversial (6), EVAR carries many benefits, especially those attributable to minivasive approach and clinical effectiveness in the short- and mid-term term $(7,8)$. Nevertheless, some complications have been shown to influence the outcome of patients undergoing EVAR. In particular, endoleaks and graft thrombosis are relatively frequent complications, both necessitating accurate radiological monitoring and possible reintervention $(9,10)$. Graft infection is also a rare but lifethreatening event after EVAR, whose surgical treatment requires high technical skills (11). Finally, an unexpected systemic inflammatory response, conventionally known as "post-implantation syndrome" (PiS), may occur several hours or days after EVAR. This condition is typically characterized by fever, leucocytosis and, occasionally, by coagulation abnormalities $(12,13)$. Although this serious complication has been known for quite a long time, its pathogenetic mechanisms and clinical relevance are still debated. In most cases PiS develops as a transient systemic inflammatory response, with no major adverse consequences for the patients. Nevertheless, some severe cases have also been described, in whom serious clinical signs develop, up to onset of severe systemic inflammatory response syndrome
(SIRS) and multi-system organ failure (MOF), and who shall hence necessitate aggressive medical treatment and prolonged hospital stay (13). Several laboratory biomarkers have been measured in the attempt of timely predicting PiS, especially C reactive protein (CRP), interleukin (IL)-6 and IL-8, but none of these was found to have a satisfactory prognostic performance $(14,15)$.

The red blood cell distribution width (RDW), an easy and simple parameter which can now be measured by the vast majority of modern hematological analyzers (16), reflects the presence of anisocytosis, i.e., the variability of erythrocyte volumes, expressed in percentage, as the ratio between the standard deviation of mean corpuscular volume (MCV) and the value of MCV. Several lines of evidence garnered over the past decade suggest that RDW is a valuable diagnostic and prognostic biomarker in patients with many cardiovascular disorders (17). More recently, Güngör et al. showed that an increased value of RDW displayed good performance for diagnosing AAA, thus suggesting that its measurement may provide useful information for the early management of this condition (18). Nevertheless, no information has been published so far on the prognostic performance of RDW after surgical management of AAA, especially for predicting the development of PiS after EVAR. Therefore, we planned this observational, retrospective study in order to evaluate the potential role of RDW in identifying AAA patients at greater risk of developing PiS after EVAR.

\section{Methods}

\section{Study population}

The study population consisted of all patients undergoing elective EVAR for infrarenal AAA at the Department of Vascular Surgery (n=124), University Hospital of Verona (Verona, Italy), during a 2-year period (i.e., between June 1, 2016 and May 31, 2018). An updated prospectively held database was used for data collection. The dedicated database included all demographical information, relevant cardiovascular risk factors, anatomic morphology, operative features along with procedural time, hospitalization length, and follow up. Anatomical data included distal aortic bifurcation diameter, common iliac arteries width and aneurysm related measurements (maximum sack diameter, proximal and distal neck evaluation). Inclusion criteria were patients with suitable anatomy for standard EVAR affected by asymptomatic infrarenal AAA according to the clinical practice guidelines 
of European Society for Vascular Surgery (19). We excluded patients with ruptured or symptomatic aneurysm, and those necessitating maneuvers during endovascular procedures (Chimney, Iliac branches, Snorkel, Endoanchors).

The endovascular procedure was performed according to the personal practice of the skilled vascular surgeon present at the operating room. Graft size was chosen according on preoperative planning, and graft oversize was calculated between $15-20 \%$ of proximal aortic diameter, accordingly to the Instructions for Use (IFU). Local anaesthesia and percutaneous approach were performed whenever possible. A standard dose of heparin was administered during the surgery (100 UI/kg). Early surgical outcomes were classified according to the current reporting standards for EVAR.

Technical success of the procedure was defined as completion of EVAR with evidence of patent graft without significant twist, kinks or obstruction ( $>30 \%$ luminal stenosis or a pressure gradient $>10 \mathrm{mmHg}$ ) and absence of either a type I or III endoleak. After the procedure, all patients maintained their preoperative oral therapy and received a daily dose of antiplatelet or anticoagulant agents, which were continued indefinitely after discharge. All patients underwent clinical and radiological (abdominal ultrasound and CT-scan) follow up after one month of surgery.

\section{Laboratory testing}

Hematological testing, which included the assessment of hemoglobin, $\mathrm{MCV}$ and RDW, was carried out on $\mathrm{K}_{2} \mathrm{EDTA}$ (ethylenediaminetetraacetic acid) anticoagulated blood, using the same Sysmex XN instrumentation (Sysmex $\mathrm{XN}$, Sysmex Inc., Kobe, Japan) throughout the study period. Blood samples were collected at patient admission $(n=124)$ and the day after surgery $(n=124)$. The mean inter-assay imprecision of hemoglobin, MCV and RDW on this analyzer has been earlier reported to be $1.1 \%$, $0.9 \%$ and $1.3 \%$, respectively (20). The local laboratory is certified according to the ISO 15189:2012 standard, and the quality of test results has hence been validated during the study by regular performance of internal quality control (IQC) procedures and participation to an external quality assessment (EQA) scheme. The delta variation of hemoglobin, MCV and RDW was calculated as the ratio between the value measured the day after surgery and that obtained at patient admission [i.e., (postoperative value)/ (preoperative value)].

\section{Statistical analysis}

Significance of differences among groups was assessed with Mann-Whitney (for continuous variables) or Chisquare with Yates correction (for categorical variables) tests. Independent significant associations with the primary endpoint (i.e., development of PiS) were identified by multivariable linear regression analysis, in which PiS onset was entered as dependent variable, whilst sex, age, delta hemoglobin, delta MCV and delta RDW were entered as independent variables. The further risk of developing postoperative fever was expressed as odds ratio (OR) and the corresponding $95 \%$ confidence interval (95\% CI). The diagnostic performance was finally evaluated using Receiver Operating Characteristics (ROC) curve analysis, with calculation of the area under the curve (AUC). The statistical analysis was performed with Analyse-it (Analyseit Software Ltd, Leeds, UK) and MedCalc Version 12.3.0 (MedCalc Software, Mariakerke, Belgium). Statistical significance was set at $\mathrm{P}<0.05$.

This retrospective observational study was based on anonymized patients' data, was carried out in accordance with the Declaration of Helsinki, under the terms of relevant local legislation, and was cleared by the Institutional Review Board. The requirement for informed consent was waived due to the observational, retrospective nature of this study.

\section{Results}

The final study population consisted of 124 patients (10 women and 114 men; median age, 75 years and interquartile range, 8 years). The mean procedural time was $68 \pm 13$ min (range, 42-162 min). Procedural success was $100 \%$, with no cases of perioperative mortality. No blood transfusion was necessary before discharge and no other complications were observed during hospital stay. The mean hospital stay was 1.3 days.

In agreement with the frequency earlier reported by others (14), 55 patients (i.e., 44\%) developed PiS (mean period of onset, 1 day; IQR, 1 day), whilst revascularization failure only occurred in two patients (i.e., 1.6\%). The main results of this study are shown in Table 1. In both categories of patients who developed or not PiS, the hemoglobin values significantly decreased after surgery, by approximately $15 \%$. An opposite trend was noticed for MCV, although the increase did not reach statistical significance in patients 
Table 1 Demographic, clinical and hematological data in patients with or without post-implantation syndrome (PiS) after endovascular aneurysm repair for abdominal aortic aneurism

\begin{tabular}{|c|c|c|c|c|c|c|}
\hline Variable & \multicolumn{3}{|c|}{ No PiS (n=69) } & \multicolumn{3}{|c|}{ PiS $(n=55)$} \\
\hline Hemoglobin $(\mathrm{g} / \mathrm{L})$ & 144 [18] & 122 [22] & $<0.001$ & $144[21]$ & 117 [25] & $<0.001$ \\
\hline MCV (fL) & $91.4(7.4)$ & $90.9(6.6)$ & $<0.001$ & $93.0(7.3)$ & $92.3(6.3)$ & 0.069 \\
\hline RDW (\%) & $14.2(2.0)$ & $14.0(1.7)$ & 0.022 & $13.9(1.4)$ & $14.2(2.1)$ & 0.002 \\
\hline
\end{tabular}

Data are shown as median and interquartile range. EVAR, endovascular aneurysm repair; MCV, mean corpuscular volume; RDW, red blood cell distribution width.

Table 2 Demographic, clinical and hematological data in patients with or without post-implantation syndrome (PiS) after endovascular aneurysm repair for abdominal aortic aneurism

\begin{tabular}{lccc}
\hline Variable & No PiS $(\mathrm{n}=69)$ & $\mathrm{PiS}(\mathrm{n}=55)$ & $\mathrm{P}$ \\
\hline Age (years) & $77[9]$ & $74[8]$ & 0.063 \\
Sex (F/M) & $6 / 63$ & $4 / 51$ & 0.386 \\
Hypertension (n) & $68(98.6)$ & $54(98.2)$ & 0.436 \\
History of CAD (n) & $17(24.6)$ & $25(45.5)$ & 0.012 \\
Diabetes (n) & $6(8.7)$ & $8(14.5)$ & 0.231 \\
Chronic renal failure (n) & $10(14.5)$ & $10(18.2)$ & 0.379 \\
Antiplatelet treatment & $61(88.4)$ & $51(92.7)$ & 0.308 \\
Oral anticoagulant treatment & $8(11.6)$ & $4(7.3)$ & 0.308 \\
Delta hemoglobin & $0.85[11]$ & $0.86[0.09]$ & 0.221 \\
Delta MCV & $0.99[0.03]$ & $0.99[0.03]$ & 0.175 \\
RDW & & & \\
Delta value & $0.99[0.04]$ & $1.02[0.05]$ & $<0.001$ \\
Delta value $>1$ (n) & $24(34.8)$ & $34(61.8)$ & 0.002 \\
\hline
\end{tabular}

Data are shown as median and interquartile range [IQR] or number and percentage. CAD, coronary artery disease; MCV, mean corpuscular volume; RDW, red blood cell distribution width.

who developed PiS. The RDW value displayed an opposed trend in patients who developed PiS than in those who did not, wherein it was found to be significantly increased in the former cohort and it was marginally but significantly decreased in the latter (Table 1). Age and sex distributions were found to be similar in patients who developed PiS or not, as shown in Table 2. Patients who developed PiS were also more likely to have a positive history of coronary artery disease (CAD; $45.5 \%$ vs. $24.6 \%, \mathrm{P}=0.012)$, but not of hypertension (98.2 vs. 98.6\%; $\mathrm{P}=0.436)$, diabetes $(14.5 \%$ vs. 8.7\%; $\mathrm{P}=0.231)$, chronic renal failure $(18.2 \%$ vs. $14.5 \%$; $\mathrm{P}=0.379)$, and of receiving antiplatelet $(92.7 \%$ vs. $88.4 \%$; $\mathrm{P}=0.308)$ or oral anticoagulant $(7.3 \%$ vs. $11.6 \% ; \mathrm{P}=0.308)$ therapy immediately after EVAR (Table 2).

Although the delta variation of hemoglobin and MCV did not reach statistical significance by comparing patients who developed PiS with those who did not, delta RDW was found to be significantly higher in the former cohort (Table 2). Accordingly, the rate of patients with delta RDW $>1$ was also significantly higher in patients who developed PiS that in those who did not $(61.8 \%$ vs. $34.8 \% ; \mathrm{P}=0.002)$. Unlike age ( $\beta$ coefficient, $-0.005 ; 95 \%$ CI, -0.018 to 0.008 ; $\mathrm{P}=0.406$ ), sex ( $\beta$ coefficient, $-0.028 ; 95 \% \mathrm{CI},-0.347$ to 0.299; $\mathrm{P}=0.860)$, delta hemoglobin ( $\beta$ coefficient, -0.381 ; $95 \% \mathrm{CI},-1.329$ to $0.567 ; \mathrm{P}=0.428)$ and delta $\mathrm{MCV}$ ( $\beta$ coefficient, 2.422; 95\% CI, -1.106 to 5.949; $\mathrm{P}=0.177$ ), delta RDW remained independently associated with development of PiS ( $\beta$ coefficient, 2.023; 95\% CI, 0.8303.216; $\mathrm{P}=0.001)$ in multivariate linear regression analysis. A highly significant inverse correlation was also noted between the time of PiS onset and delta RDW (Spearman's correlation, $-0.37 ; 95 \% \mathrm{CI},-0.58$ to $-0.11 ; \mathrm{P}=0.006$ ). Overall, a delta RDW $>1$ after EVAR was associated with over 3-fold enhanced risk of developing PiS (OR, 3.04; 95\% CI, 1.45-6.34; $\mathrm{P}=0.003)$. In ROC curve analysis, delta RDW exhibited a good diagnostic performance for predicting the development of PiS (AUC, 0.69; 95\% CI, 0.60-0.79; $\mathrm{P}<0.001$ ) (Figure 1). A delta RDW >1 was characterized by 0.70 negative predictive value and 0.56 positive predictive value for predicting $\mathrm{PiS}$ onset.

\section{Discussion}

Albeit EVAR is now regarded as the reference approach for managing patients with symptomatic and large AAAs, this endovascular procedure carries specific risks, including 


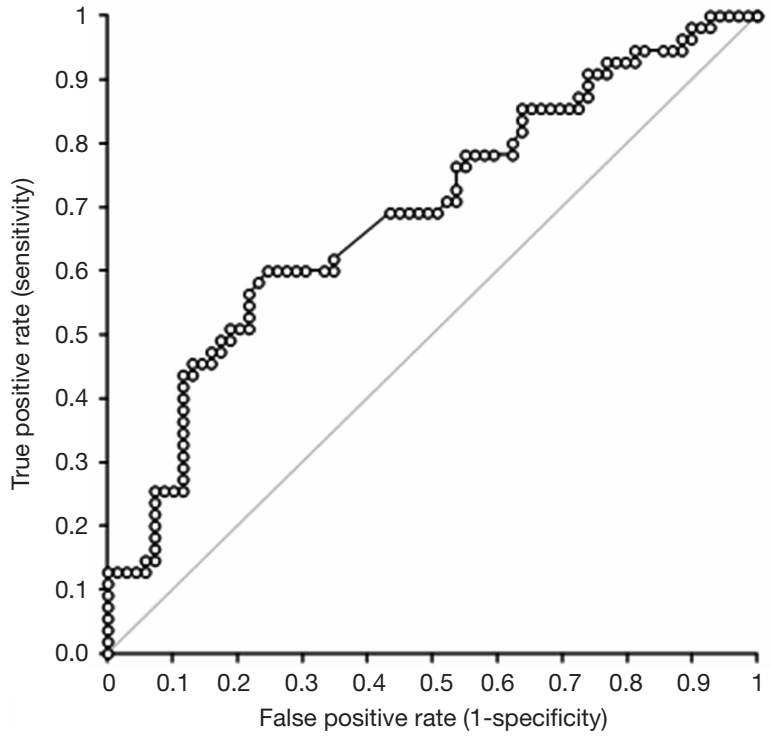

Figure 1 Receiver operating characteristic (ROC) curve analysis of delta red blood cell distribution width (RDW) for predicting the development of post-implantation syndrome (PiS) after endovascular aneurysm repair for abdominal aortic aneurism.

the possible development of PiS. Some demographic and clinical factors have been earlier identified for predicting the risk of complications after EVAR, including older age, female sex, renal impairment, congestive heart failure, pulmonary dysfunction and electrocardiographic evidence of ischemia (21). However, no single laboratory parameter, among those which have been investigated so far, has seemly displayed such an efficient diagnostic performance to be routinely used for risk stratification (21).

The results of this observational, retrospective study convincingly demonstrate that an increase of RDW value after EVAR is a strong and independent predictor of developing PiS, thus reinforcing previous evidence that this simple and relative inexpensive parameter displays good performance for diagnosing AAA (18). Interestingly, the highly significant inverse correlation observed between delta RDW and time of PiS onset after surgery (Spearman's correlation, $-0.37 ; \mathrm{P}=0.006$ ) underscores also that the larger is the increase of RDW after surgery, the more likely is the early development of this complication. In fact, when the statistical analysis was limited to patients with early development of PiS (i.e., $\leq 1$ day; $n=24$ ), the association between delta RDW $>1$ and the risk of PiS was over twofold higher (OR, 7.13; 95\% CI, 2.37-21.46; $\mathrm{P}<0.001)$ compared to the entire cohort of patients (i.e., OR, 3.04). Altogether these findings would hence confirm that anisocytosis is strongly linked with the pathogenesis of inflammation (22), and support the hypothesis that measuring RDW before and after EVAR, for calculating the relative delta variation EVAR, may be a valuable prognostic tool for stratifying the immediate postoperative risk of developing PiS in AAA patients needing endovascular management. Interestingly, similar findings have been earlier reported by Aung et al. (23), who showed that a gradual increase of RDW during hospitalization was associated with enhanced risk of death in patients undergoing transcatheter aortic valve implantation. von Meijenfeldt et al. (24) also concluded that an increased RDW value at hospital discharge was a strong and independent predictor of death and readmission in critically ill patients undergoing vascular surgery.

Whether higher anisocytosis should then be considered an active player or a simple bystander in the pathogenesis of PiS after EVAR remains to be unravelled. Indeed, anisocytosis often develops as a consequence of endothelial damage and failure of vascular repair (25), oxidative (26) or surgical stress (27), and endovascular thrombosis (28), all conditions which may then promote, or even directly trigger, the release of proinflammatory mediators that are actively involved in the pathogenesis of the inflammatory response characterizing $\mathrm{PiS}(12,29)$ and other severe conditions (30).

On the other hand, it is also noteworthy mentioning here that ongoing anisocytosis, as mirrored by an increase of RDW value after EVAR, may play a direct role in pathogenesis of PiS. Recent evidence attest that increased RDW is paralleled by lower erythrocyte deformability, which would then impair blood flow and contribute to tissue ischemia (31), but also by an increased propensity of developing both arterial and venous thrombosis (32). Anisocytosis is also strongly associated with the severity of underlying atherosclerotic disease (33), and with failure of arterial revascularization surgery (34), thus ultimately promoting or directly triggering the development of postEVAR complications, including PiS.

\section{Conclusions}

The results of this retrospective, observational study suggest that the measurement of RDW before and after EVAR, may represent an efficient prognostic tool for stratifying the risk of developing PiS in patients with AAA, especially in the early postoperative period. 


\section{Acknowledgements}

None.

\section{Footnote}

Conflicts of Interest: The authors have no conflicts of interest to declare.

Ethical Statement: This retrospective observational study was based on anonymized patients' data, was carried out in accordance with the Declaration of Helsinki, under the terms of relevant local legislation, and was cleared by the Institutional Review Board. The requirement for informed consent was waived due to the observational, retrospective nature of this study.

\section{References}

1. Aggarwal S, Qamar A, Sharma V, et al. Abdominal aortic aneurysm: A comprehensive review. Exp Clin Cardiol 2011;16:11-5.

2. Kumar Y, Hooda K, Li S, et al. Abdominal aortic aneurysm: pictorial review of common appearances and complications. Ann Transl Med 2017;5:256.

3. McPhee JT, Hill JS, Eslami MH. The impact of gender on presentation, therapy, and mortality of abdominal aortic aneurysm in the United States, 2001-2004. J Vasc Surg 2007;45:891-9.

4. Murana G, Alfonsi J, Di Marco L, et al. Open thoracoabdominal aortic aneurysm repair in expert hands: "all roads lead to Rome". Ann Transl Med 2017;5:414.

5. Patel R, Sweeting MJ, Powell JT, et al. Endovascular versus open repair of abdominal aortic aneurysm in 15-years' follow-up of the UK endovascular aneurysm repair trial 1 (EVAR trial 1): a randomised controlled trial. Lancet 2016;388:2366-74.

6. Dua A, Kuy S, Lee CJ, et al. Epidemiology of aortic aneurysm repair in the United States from 2000 to 2010. J Vasc Surg 2014;59:1512-7.

7. Park BD, Azefor NM, Huang CC, et al. Elective endovascular aneurysm repair in the elderly: trends and outcomes from the Nationwide Inpatient Sample. Ann Vasc Surg 2014;28:798-807.

8. Zhou W, Blay E Jr, Varu V, et al. Outcome and clinical significance of delayed endoleaks after endovascular aneurysm repair. J Vasc Surg 2014;59:915-20.

9. Cochennec F, Becquemin JP, Desgranges P, et al. Limb graft occlusion following EVAR: clinical pattern, outcomes and predictive factors of occurrence. Eur J Vasc Endovasc Surg 2007;34:59-65.

10. Capoccia L, Speziale F, Menna D, et al. Preliminary Results from a National Enquiry of Infection in Abdominal Aortic Endovascular Repair (Registry of Infection in EVAR--R.I.EVAR). Ann Vasc Surg 2016;30:198-204.

11. Arnaoutoglou E, Papas N, Milionis H, et al. Postimplantation syndrome after endovascular repair of aortic aneurysms: need for postdischarge surveillance. Interact Cardiovasc Thorac Surg 2010;11:449-54.

12. Velázquez OC, Carpenter JP, Baum RA, et al. Perigraft air, fever, and leukocytosis after endovascular repair of abdominal aortic aneurysms. Am J Surg 1999;178:185-9.

13. Chang CK, Chuter TA, Niemann CU, et al. Systemic inflammation, coagulopathy, and acute renal insufficiency following endovascular thoracoabdominal aortic aneurysm repair. J Vasc Surg 2009;49:1140-6.

14. Tsilimigras DI, Sigala F, Karaolanis G, et al. Cytokines as biomarkers of inflammatory response after open versus endovascular repair of abdominal aortic aneurysms: a systematic review. Acta Pharmacol Sin 2018;39:1164-75.

15. Arnaoutoglou E, Kouvelos G, Papa N, et al. Prospective evaluation of post-implantation inflammatory response after EVAR for AAA: influence on patients' 30 day outcome. Eur J Vasc Endovasc Surg 2015;49:175-83.

16. Salvagno GL, Sanchis-Gomar F, Picanza A, et al. Red blood cell distribution width: A simple parameter with multiple clinical applications. Crit Rev Clin Lab Sci 2015;52:86-105.

17. Danese E, Lippi G, Montagnana M. Red blood cell distribution width and cardiovascular diseases. J Thorac Dis 2015;7:E402-11.

18. Güngör B, Ozcan KS, Ozpamuk Karadeniz F et al. Red cell distribution width is increased in patients with ascending aortic dilatation. Turk Kardiyol Dern Ars 2014;42:227-35.

19. Moll FL, Powell JT, Fraedrich G, et al. Management of abdominal aortic aneurysms clinical practice guidelines of the European society for vascular surgery. Eur J Vasc Endovasc Surg 2011;41 Suppl 1:S1-58.

20. Birindelli S, Aloisio E, Carnevale A, et al. Evaluation of long-term imprecision of automated complete blood cell count on the Sysmex XN-9000 system. Clin Chem Lab Med 2017;55:e219-22.

21. Al-Omran M, Verma S, Lindsay TF, et al. Clinical decision making for endovascular repair of abdominal aortic aneurysm. Circulation 2004;110:e517-23. 
22. Lippi G, Targher G, Montagnana M, et al. Relation between red blood cell distribution width and inflammatory biomarkers in a large cohort of unselected outpatients. Arch Pathol Lab Med 2009;133:628-32.

23. Aung N, Dworakowski R, Byrne J et al. Progressive rise in red cell distribution width is associated with poor outcome after transcatheter aortic valve implantation. Heart 2013;99:1261-6.

24. von Meijenfeldt GCI, van der Laan MJ, Zeebregts CJAM et al. Red cell distribution width at hospital discharge and out-of hospital outcomes in critically ill non-cardiac vascular surgery patients. PLoS One 2018;13:e0199654.

25. Rodríguez-Carrio J, Alperi-López M, López P, et al. Red cell distribution width is associated with endothelial progenitor cell depletion and vascular-related mediators in rheumatoid arthritis. Atherosclerosis 2015;240:131-6.

26. Semba RD, Patel KV, Ferrucci L, et al. Serum antioxidants and inflammation predict red cell distribution width in older women: the Women's Health and Aging Study I. Clin Nutr 2010;29:600-4.

27. Yazıcı P, Demir U, Bozdağ E, et al. What is the effect of treatment modality on red blood cell distribution width in patients with acute cholecystitis? Ulus Cerrahi Derg 2015;31:1-4.

Cite this article as: Veraldi GF, Mezzetto L, Scorsone L, Macrì M, Bovo C, Simoncini F, Criscenti P, Lippi G. Red blood cell distribution width (RDW) is an independent predictor of postimplantation syndrome in patients undergoing endovascular aortic repair for abdominal aortic aneurysm. Ann Transl Med 2018;6(23):453. doi: 10.21037/atm.2018.11.07
28. Bojakowski K, Dzabic M, Kurzejamska E, et al. A high red blood cell distribution width predicts failure of arteriovenous fistula. PLoS One 2012;7:e36482.

29. Yao YT, Li LH, Lei Q, et al. Noninfectious fever following aortic surgery: incidence, risk factors, and outcomes. Chin Med Sci J 2009;24:213-9.

30. Seth HS, Mishra P, Khandekar JV, et al. Relationship between High Red Cell Distribution Width and Systemic Inflammatory Response Syndrome after Extracorporeal Circulation. Braz J Cardiovasc Surg 2017;32:288-94.

31. Patel KV, Mohanty JG, Kanapuru B, et al. Association of the red cell distribution width with red blood cell deformability. Adv Exp Med Biol 2013;765:211-6.

32. Chesnutt JK, Han HC. Effect of Red Blood Cells on Platelet Activation and Thrombus Formation in Tortuous Arterioles. Front Bioeng Biotechnol 2013;1:18.

33. Erdem A, Ceylan US, Esen A, et al. Clinical usefulness of red cell distribution width to angiographic severity and coronary stent thrombosis. Int J Gen Med 2016;9:319-24.

34. Tunçez A, Çetin MS, Çetin EH, et al. Association between RDW and stent thrombosis in patients with ST-elevation myocardial infarction undergoing primary percutaneous coronary intervention. Medicine (Baltimore) 2017;96:e5986. 